\title{
Dual-Energy CT zur postoperativen Langzeitkontrolle nach endovaskulär therapierten abdominellen Aortenaneurysmen
}

\author{
Dual-Energy CT in the Follow-Up after Endovascular Abdominal Aortic Aneurysm \\ Repair
}

Autoren

Institute
A. Brägelmann ${ }^{1}$, A. Bunck ${ }^{1,2}$, K. Donas ${ }^{3}$, B. Kasprzak ${ }^{3}$, D. Maintz ${ }^{1,2}$, W. Heindel ${ }^{1}$, H. Seifarth ${ }^{1}$

Institut für Klinische Radiologie, Universitätsklinikum Münster

2 Institut und Poliklinik für Radiologische Diagnostik, Universitätsklinikum Köln

Klinik für Vaskuläre und Endovaskuläre Chirurgie, Universitätsklinikum Münster

\author{
Key words \\ - aorta \\ - CT angiography \\ - aneurysm
}

eingereicht 8.8 .2012

akzeptiert 28.11 .2012

Bibliografie

Dol http://dx.doi.org/

10.1055/s-0032-1330447

Online-Publikation: 20.2.2013

Fortschr Röntgenstr 2013; 185 :

351-357 @ Georg Thieme

Verlag KG Stuttgart · New York ·

ISSN 1438-9029

Korrespondenzadresse PD Dr. Harald Seifarth Institut für Klinische Radiologie, Universitätsklinikum Münster Albert-Schweitzer-Campus 1 48149 Münster

seifarth@uni-muenster.de

Tel.: ++49/251/8347310

Fax: ++49/2 51/8349656

\section{Zusammenfassung \\ $\nabla$}

Ziel: Ziel dieser Studie war es, zu ermitteln, ob in der postoperativen CT-Nachsorge nach endovaskulär therapierten abdominellen Aortenaneurysmen (EVAR) die Strahlenexposition durch virtuelle Nativbilder aus der Dual Energy CT reduziert werden kann. Darüber hinaus sollte untersucht werden, ob die Darstellung von Endolecks aufgrund der relativen Kontrastanhebung bei niedrigen Röhrenspannungen in der venösen Phase ähnlich gut gelingt wie in der arteriellen Phase. Material und Methoden: Zwischen Februar 2009 und März 2010 wurden bei 47 Patienten 51 CT-Untersuchungen durchgeführt, bestehend aus Nativscan und arterieller und venöser Phase im DualEnergy-Verfahren (Protokoll A, Referenzstandard). Aus den Datensätzen der venösen Phase wurden virtuelle Bilder rekonstruiert. In Protokoll B wurden reelle Nativbilder durch virtuelle ersetzt, Protokoll $\mathrm{C}$ beinhaltete virtuelle Nativbilder und $80 \mathrm{kV}$-Bilder der venösen Phase. Alle Daten wurden anonymisiert und von zwei unabhängigen Radiologen ausgewertet. Sensitivität, Spezifität, negativ und positiv prädiktiver Wert wurden für Protokoll C berechnet. Für jeden Scan wurde die effektive Dosis ermittelt.

Ergebnisse: Alle in Protokoll A identifizierten Endolecks wurden auch in Protokoll B und C erkannt. Für Protokoll C lagen Sensitivität und negativ prädiktiver Wert bei $100 \%$, Spezifität bei $94,1 \%$ und positiv prädiktiver Wert bei 89,5\%. Protokoll C führte zu einer Reduktion der Strahlenexposition um 62,45\%. Schlussfolgerung: Ein Scanprotokoll aus virtuellen Nativbildern und 80kV-Bildern der venösen Phase stellt eine zuverlässige Alternative zur Diagnose von Endolecks nach EVAR dar, bei einer Strahlenreduktion gegenüber dem Standardverfahren um $62,45 \%$.

\section{Abstract \\ $\nabla$}

Purpose: This study investigates the dual-energy procedure for postoperative CT follow-up scans after endovascularly treated abdominal aortic aneurysms. The procedure is analyzed with respect to its sensitivity and specificity as well as the associated radiation exposure.

Materials and Methods: 51 examinations were carried out on 47 patients between February 2009 and March 2010. For each patient, a non-enhanced, an arterial and a venous scan were conducted, the latter two using the dual-energy technology. Virtual images for the non-enhanced phase were reconstructed from the data taken in the venous phase. Protocol A, the reference standard, consisted of non-enhanced images and images of the arterial and venous phase. In protocol $\mathrm{B}$, standard non-enhanced images were replaced by the reconstructed virtual non-enhanced images. Protocol C consisted only of virtual non-enhanced and $80 \mathrm{kV}$ images taken during the venous phase. All data was anonymized and evaluated by two independent radiologists. For protocol C, sensitivity, specificity, negative and positive predictive values were computed. The effective radiation dosage was determined for each scan.

Results: All endoleaks identified in protocol A were found using protocols B and C. For protocol $\mathrm{C}$, the sensitivity and negative predictive value were $100 \%$, the specificity was $94.1 \%$, and the positive predictive value was $89.5 \%$. Compared to protocol A, protocol $\mathrm{C}$ reduces the radiation exposure by $62.45 \%$.

Conclusion: A scan protocol consisting of virtual non-enhanced images as well as $80 \mathrm{kV}$ images taken during the venous phase was found to be a reliable alternative method for diagnosing endoleaks, while reducing the radiation exposure by $62.45 \%$. 


\section{Einleitung}

$\nabla$

Die Therapie der abdominellen Aortenaneurysmen (AAA) hat sich im Laufe der vergangenen 10 Jahre nachhaltig verändert. Lange Zeit galt die offene Operation als Standardtherapieverfahren. 1991 stellten Parodi et al. das Prinzip der endovaskulären Implantation endoluminaler Stentprothesen (EVAR - endovascular aortic repair) vor [1], welches heute als gering invasives Verfahren immer mehr Anwendung findet. Trotz einer hohen intraoperativen Erfolgsrate von über $98 \%$ tritt innerhalb von 10 Jahren nach Stentimplantation bei 25 - 50\% der Patienten eine interventionsbedürftige Komplikation auf [2-4]. Hieraus leitet sich die Notwendigkeit einer lebenslangen postinterventionellen Nachsorge inklusive Bildgebung ab [5]. Endolecks, d. h. persistierender Blutfluss im Aneurysmasack, stellen mit einer Inzidenz von ca. $50 \%$ die häufigste Komplikation dar [2]. Diese Endolecks können zu einer Zunahme des Aneurysmadurchmessers führen, eine Therapie ist jedoch nur in ca. einem Drittel der Fälle notwendig [6].

Zur sicheren Erkennung und Klassifikation von Endolecks hat sich die kontrastmittelverstärkte Multidetektor-Computertomografie als zuverlässige Untersuchungsmethode etabliert [7-9]. Dabei stellt die Erfassung von nativen, arteriellen und venösen CT-Scans das Standardverfahren dar [10]. Die native Serie als Ergänzung zur kontrastmittelverstärkten Serie dient hier zur sicheren Differenzierung von Verkalkungen und Kontrastmittel im Endoleck. Dieses Vorgehen führt jedoch zu einer erheblichen Strahlenbelastung der Patienten [11].

Die 2005 vorgestellte Dual-Energy-Dual-Source-Computertomografie (DSCT) erlaubt die Verwendung unterschiedlicher Röhrenspannungen für die beiden im CT verbauten Röhren. Die unterschiedliche Schwächung der Röntgenstrahlen durch das Kontrastmittel bei verschiedenen Spektren ermöglicht eine Differenzierung kontrastmittelhaltiger Strukturen und die Berechnung sogenannter virtueller Nativbilder [12, 13]. Darüber hinaus kommt es zu einer relativen Dichteanhebung kontrastmittelhaltiger Strukturen im Niedrigenergie-Datensatz [14].

Ziel dieser Studie war es, zu ermitteln, ob die native Phase zur Differenzierung von Kalzifikationen und Endolecks in der Nachsorge nach EVAR durch die Berechnung virtueller Nativbilder ersetzt werden kann. Darüber hinaus sollte untersucht werden, ob Endolecks im Niedrigenergiedatensatz der venösen Phase mit ausreichender Sicherheit erkannt werden können.

\section{Material und Methoden}

\section{Patienten}

Die Studie wurde durch die Ethikkommission bewilligt, von allen Patienten wurde nach ausführlicher Aufklärung eine schriftliche Zustimmung eingeholt.

Zwischen Februar 2009 und März 2010 wurden 47 Patienten (1 Frau, 46 Männer) nach endovaskulärer Therapie eines abdominellen Aortenaneurysmas in die Studie aufgenommen und im Rahmen der Nachsorge untersucht. Die demografischen Daten sind aus Tab. 1 ersichtlich. Bei 4 Patienten wurden während des Auswertungszeitraums 2 Untersuchungen durchgeführt, sodass insgesamt 51 Untersuchungen für die Studie ausgewertet wurden. Der Untersuchungszeitpunkt lag bei 23 Patienten innerhalb der ersten Woche nach EVAR, bei 8 Patienten zwischen 1 und 9 Monaten nach Stentimplantation. Bei 20 Patienten wurde die Untersuchung nach mehr als 12 Monaten als Langzeitkontrol- le durchgeführt. Bei 25 Patienten wurde zur Therapie des Aortenaneurysmas ein Zenith-Stentgraft (Cook, Limerick, Irland), bei 5 Patienten Talent-Stentgrafts und bei 14 Patienten EndurantStentgrafts (jeweils von Medtronic, Tolochenaz, Schweiz) zur Aneurysmaausschaltung verwendet. Bei drei ex-domo behandelten Patienten lagen keine Daten zum verwendeten Stent vor.

\section{Bildgebung}

Alle Untersuchungen wurden mit einem Dual-Source-CT (Somatom Definition; Siemens Medical Solutions, Forchheim, Deutschland) durchgeführt. Bei jedem Patienten wurde die Bauchaorta in kraniokaudaler Richtung bis zur Iliakalbifurkation jeweils dreimal dargestellt: Nativtechnik, arterielle und venöse Phase, wobei die Datensätze der arteriellen und venösen Phase mittels DualEnergy-Verfahren erstellt wurden. Die jeweiligen Untersuchungsparameter sind aus $\bullet$ Tab. 2 ersichtlich.

Zur i. v. Kontrastierung wurden $120 \mathrm{ml}$ nicht ionisches Kontrastmittel (370 mg Iod $/ \mathrm{ml}$, Ultravist ${ }^{\circledR}$ Bayer HealthCare, Berlin, Deutschland) mit einer Flussrate von $5 \mathrm{ml} / \mathrm{s}$ (entsprechend 1,85 g Iod/s) über eine Antekubitalvene appliziert. Das Start-Delay für die arterielle Phase wurde mittels Bolustracking festgelegt. Hierzu wurde eine Region of Interest (ROI) auf Höhe der Nierenarterien in die Aorta abdominalis platziert. Die Untersuchung wurde 6 s nach Erreichen einer Dichte von 140 HU innerhalb der ROI gestartet. Für die venöse Phase wurde ein Delay von 60 s gewählt.

\section{Dual-Energy-Nachbearbeitung}

Um einen arteriellen und venösen Referenzstandard zu erhalten, wurde aus den jeweiligen $80 \mathrm{kV}$ - und $140 \mathrm{kV}$-Daten zunächst unter Verwendung folgender Formel $\left(H U_{\text {fused }}=0,3 \times H U_{80 \mathrm{kV}}+(1-\right.$ $\left.0,3) \times H U_{140 \mathrm{kV}}\right)$ ein Mischbild berechnet. Die Dichtewerte und

Tab. 1 Patientendaten und Häufigkeiten der Endoleck-Typen.

\begin{tabular}{|ll|}
\hline Alter (Jahre) & $73 \pm 8^{1}$ \\
\hline Männer & 46 \\
\hline Frauen & 1 \\
\hline Body-Mass-Index $\left(\mathrm{kg} / \mathrm{m}^{2}\right)$ & $27,45 \pm 3,44(22,99-35,26)^{1}$ \\
\hline Endoleck-Typ $(\mathbf{n}=\mathbf{1 7})$ & \\
\hline I & $2(11,8 \%)$ \\
\hline II & $14(82,4 \%)$ \\
\hline III & $1,0(5,9 \%)$ \\
\hline IV & 0 \\
\hline V & 0 \\
\hline${ }^{1}$ Mittelwert \pm Standardabweichung. & \\
\hline
\end{tabular}

Tab. 2 Untersuchungsparameter zur Darstellung der Bauchaorta in nativer, arterieller und venöser Phase.

\begin{tabular}{|c|c|c|}
\hline & $\begin{array}{l}\text { Nativtechnik } \\
\text { (Single Energy) }\end{array}$ & $\begin{array}{l}\text { arterielle } \& \text { venöse } \\
\text { Phase (Dual Energy) }\end{array}$ \\
\hline Kollimation (mm) & $32 \times 1,2$ & $2 \times 32 \times 0,6$ \\
\hline Pitch & 0,7 & 0,7 \\
\hline Gantry-Rotationszeit (s) & 0,5 & 0,5 \\
\hline Röhrenspannung (kV) & 120 & $\begin{array}{l}\text { Röhre A: } 140 \\
\text { Röhre B: } 80\end{array}$ \\
\hline $\begin{array}{l}\text { Strom-Zeit-Produkt } \\
(\mathrm{mAs})\end{array}$ & 160 & $\begin{array}{l}\text { Röhre A: } 49 \\
\text { Röhre B: } 270\end{array}$ \\
\hline Schichtdicke (cm) & 1,5 & 1,0 \\
\hline Inkrement (mm) & 1,0 & 0,7 \\
\hline Faltungskern & B30f & D30 f \\
\hline
\end{tabular}


Bildkontraste in diesem Datensatz entsprechen weitgehend denjenigen eines regulären, bei $120 \mathrm{kV}$ akquirierten Datensatzes [13]. Die Bilddaten wurden anschließend anonymisiert, an eine externe Workstation (Multimodality Workplace, Siemens Medical Solutions, Forchheim) übertragen und mit einer dedizierten Software (Syngo Dual Energy Version VA34) weiterverarbeitet. Zur Berechnung der virtuellen Nativbilder wurden die $80 \mathrm{kV}$ und $140 \mathrm{kV}$-Datensätze sowie die oben genannte Software genutzt. Grundsätzlich ist es möglich, Materialien durch den Einsatz verschiedener Röntgenspektren zu differenzieren, da neben der Compton-Streuung besonders der Photoeffekt von der Strahlungsenergie abhängig ist und somit unterschiedliche Strahlungsschwächungen auftreten.

Materialien mit einer hohen Kernladungszahl wie Jod zeigen bei Applikation verschiedener Strahlungsenergien deutliche Absorptionsunterschiede, was letztlich zu unterschiedlichen Dichtewerten in der CT führt [15]. Diesen Effekt kann man sich mittels eines mathematischen Algorithmus, der auf einer Drei-Material-Zerlegung basiert, zur Differenzierung von Geweben zunutze machen. Dabei wird jedes Voxel in seine 2 Hauptbestandteile Weichgewebe und Fett bzw. Jod zerlegt. Durch die Analyse der relativen Veränderungen der entsprechenden CT-Werte bei verschiedenen Spannungswerten kann das Verhältnis der Anteile von Weichgewebe zu Fett bzw. Jod bestimmt werden. Auf dieser Basis ist auch eine Subtraktion des Jodanteils möglich, wodurch virtuelle Nativbilder berechnet werden können $[13,16]$ ( Abb. 1, 2). Um eine optimale Subtraktion des Jodanteils zu erreichen, wurde für jeden Patienten im Mischbild die Kontrastmitteldichte in der Aorta kurz oberhalb des Stents mittels einer ROI-Technik bestimmt. Die gemessenen Maximalwerte wurden dann für die Berechnung des virtuellen Nativbilds verwendet. Als Minimalwert wurde die Dichte von Wasser (1 HU) verwendet. Voxel mit Dichtewerten ober- und unterhalb dieser Werte bleiben bei der Drei-MaterialZerlegung unverändert. Durch die individuellen Schwellenwerte wurde sichergestellt, dass eine optimale Subtraktion des Jodanteils erzielt wurde.

\section{Bildanalyse}

Zur Auswertung wurden die berechneten Bilddaten in 3 Auswerteprotokolle eingeteilt:

Auswerteprotokoll A umfasste die reellen Nativbilder sowie die Mischbilder der arteriellen und venösen Phase. In Protokoll B wurden die reellen Nativbilder durch virtuelle Nativbilder ersetzt. Auswerteprotokoll $\mathrm{C}$ beinhaltete wiederum die virtuellen Nativbilder aus der venösen Phase sowie die $80 \mathrm{kV}$-Bilder der venösen Phase ( Tab.3).

Die rein visuelle Auswertung erfolgte jeweils geblindet durch 2 unabhängige Radiologen. Um das Ergebnis durch Wiedererkennen der Bilder nicht zu verfälschen, fand die Beurteilung der 3 Bildserien in zufälliger Reihenfolge und in einem zeitlichen Abstand von jeweils 2 Wochen statt. Bei fehlender Übereinstimmung zwischen den Auswertern wurde ein dritter Auswerter zur Bestätigung der Diagnose hinzugezogen.

In allen 3 Auswerteprotokollen wurde die Erkennbarkeit von Endolecks untersucht. Ein Endoleck wurde dabei als Kontrastanhebung außerhalb der Stentstreben, aber innerhalb des Aneurys-

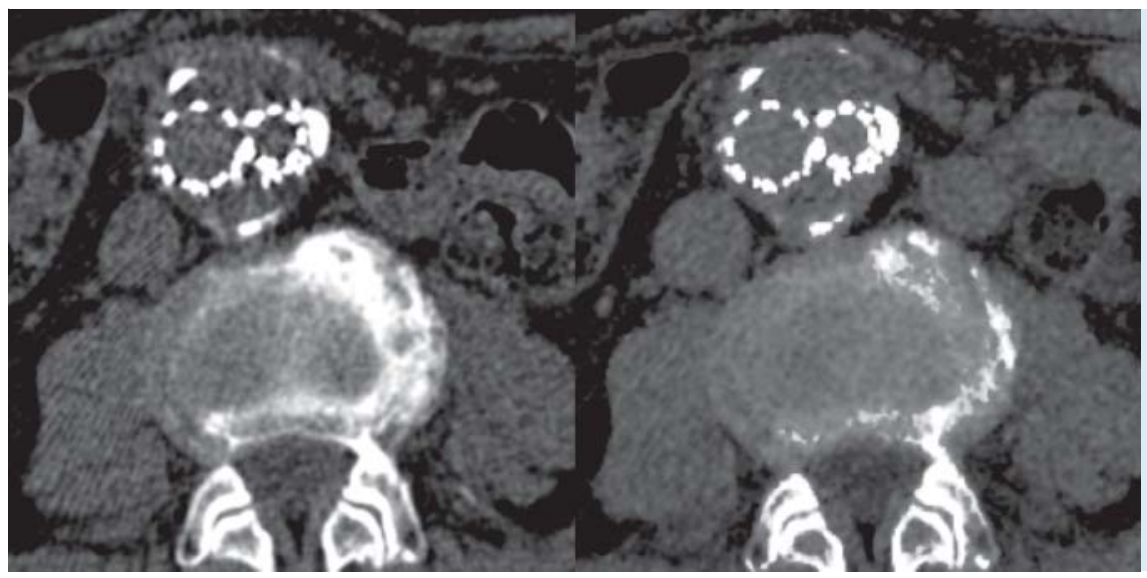

Abb. 1 Reelles Nativbild (links) und aus venösem Datensatz berechnetes virtuelles Nativbild (rechts). Die Kalzifikationen stellen sich im virtuellen Nativbild minimal kleiner dar als im tatsächlichen Nativbild. Eine Differenzierung zwischen Verkalkung und Endoleck war jedoch immer möglich.

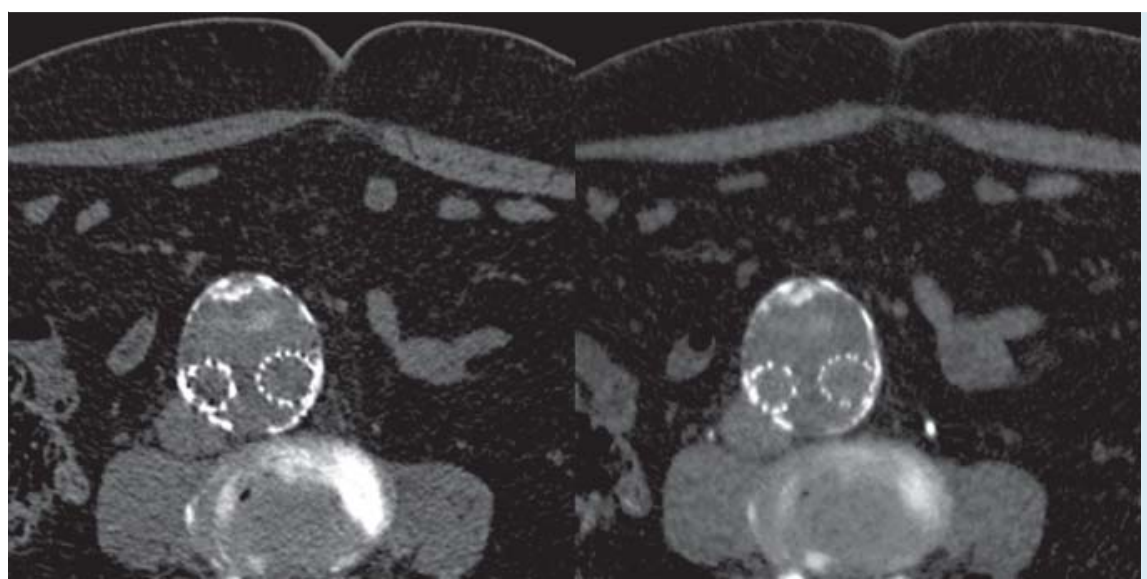

Abb. 2 Reelles Nativbild (links) und aus venösem Datensatz berechnetes virtuelles Nativbild (rechts). Die flächige Verkalkung innerhalb des Thrombus im Aneurysma stellt sich in beiden Bildern identisch dar. Die virtuellen Nativbilder ermöglichen wie die reellen Nativbilder den sicheren Ausschluss eines Endolecks. 
masacks definiert, welche in den kontrastverstärkten Bildserien, aber nicht in den nativen bzw. virtuell nativen Bildern nachweisbar war. Die Einteilung der Endolecks erfolgte nach Pitton in 5 Typen [17]. Die diagnostische Sicherheit von Diagnose oder Ausschluss eines Endolecks erfolgte anhand einer 4-stufigen LikertSkala, (1 = sicherer Ausschluss eines Endolecks, 2 und 3 = Endoleck unwahrscheinlich bzw. wahrscheinlich, $4=$ sicherer Nachweis eines Endolecks).

Zusätzlich wurde für jedes Auswerteprotokoll festgehalten ob die native Phase für die Beurteilung der Endolecks für notwendig erachtet wurde.

\section{Strahlenexposition}

Die effektive Dosis beschreibt das Ausmaß der Strahlenexposition und damit das Risiko eines biologischen Schadens für den Patienten.

Für jede Phase wurden Computertomografie-Dosis-Index (CTDIvol [mGy]), Scanlänge und Dosis-Längen-Produkt (DLP [mGy × $\mathrm{cm}])$ erfasst.

Da unser Studienkollektiv nahezu ausschließlich Männer umfasste wurde zur Berechnung der effektiven Dosis (mSv) ein Konversionskoeffizient von $\mathrm{k}=0,015(\mathrm{mSv} /[\mathrm{mGy} \times \mathrm{cm}])$ verwendet $[18,19]$.

\section{Statistische Analyse}

Das Ausmaß der Übereinstimmung zwischen den Untersuchern wurde mithilfe der Kappa-Statistik untersucht, wobei $\mathrm{k}<0$ schlechte Übereinstimmung, Werte von 0,00-0,20 geringe Übereinstimmung, 0,21 - 0,40 ausreichende Übereinstimmung, 0,41 - 0,60 mäßige Übereinstimmung, 0,61-0,80 erhebliche Übereinstimmung und 0,81-1,00 exzellente Übereinstimmung zeigen [20].

Als Referenzstandard diente Protokoll A. Wurde in Auswerteprotokoll B und C das Vorliegen eines Endolecks als wahrscheinlich oder sicher erachtet, d. h. Werte von 3 oder 4 zugeordnet, wurden diese Ergebnisse als richtig positiv $\left(r_{p}\right)$ angenommen, sofern in Protokoll A ebenfalls ein Endoleck diagnostiziert wurde. Wurde

Tab. 3 Übersicht über die verwendeten Auswerteprotokolle. Protokoll A stellt den Referenzstandard dar.

\begin{tabular}{|lllll} 
& $\begin{array}{l}\text { reelle native } \\
\text { Phase }\end{array}$ & $\begin{array}{l}\text { virtuelle } \\
\text { Nativbilder }\end{array}$ & $\begin{array}{l}\text { arterielle } \\
\text { Phase }\end{array}$ & $\begin{array}{l}\text { venöse } \\
\text { Phase }\end{array}$ \\
\hline Protokoll A & + & - & + & + \\
\hline Protokoll B & - & + & + & + \\
\hline Protokoll C & - & + & - & + \\
\hline
\end{tabular}

in Protokoll B und C kein Endoleck gefunden (Werte 1 oder 2) und entsprach dies auch den Ergebnissen aus Protokoll A, so konnte das Ergebnis als richtig negativ $\left(r_{n}\right)$ angenommen werden. Eine Diskrepanz zwischen den Ergebnissen aus den Protokollen A und B bzw. $C$ wurde als falsch negativ $\left(f_{n}\right)$ gewertet. Für die Protokolle B und C wurden jeweils Sensitivität, Spezifität, negativ und positiv prädiktive Werte für den Nachweis von Endolecks mit entsprechendem 95\%-Konfidenzintervall berechnet. Unterschiede in der Detektion von Endolecks zwischen den Gruppen wurden mittels McNemar-Test beurteilt. Unterschiede in der Häufigkeit der Heranziehung der nativen Serie zur Beurteilung der Endolecks in den verschiedenen Auswerteprotokollen wurden ebenfalls mittels NcNemar-Test beurteilt. Die mit den jeweiligen Auswertungsgruppen verbundene Strahlenexposition wurde mittels t-Test verglichen. Alle Analysen wurden mittels einer Statistiksoftware durchgeführt (SPSS Version 20; IBM Corporation, Armonk, NY).

\section{Ergebnisse}

$\nabla$

Alle 51 Untersuchungen wurden ohne Komplikationen durchgeführt und konnten in die Auswertung aufgenommen werden. Die Interobserver-Übereinstimmung in den Auswerteprotokollen A und B war sowohl hinsichtlich der Endoleck-Erkennung als auch der Klassifizierung exzellent ( $\mathrm{k}=1$ bzw. $\mathrm{k}=0,96$ ). Auch in Auswerteprotokoll $\mathrm{C}$ war die Interobserver-Übereinstimmung sowohl für die Endoleck-Detektion ( $k=0,83$ ) als auch für die Klassifizierung der Endolecks ( $\mathrm{k}=1$ ) exzellent ( $\bullet$ Abb. 3 ).

In Protokoll A wurde in 17 Fällen (33,3\%) ein Endoleck diagnostiziert, in 14 Fällen (82,4\%) lag hier ein Typ-II-Endoleck vor, in zwei Fällen $(11,8 \%)$ ein Typ-I-Endoleck und in einem Fall lag ein TypIII-Endoleck (5,9\%) vor. In einem Fall bestand kein Konsensus hinsichtlich der Klassifikation eines Endolecks. Das Endoleck wurde von einem Auswerter als Typ I, vom zweiten Auswerter als Typ III beurteilt. Ein dritter Auswerter bestätigte die Diagnose eines Typ-III-Endolecks, was auch mit der klinischen Diagnose übereinstimmte.

Die Ergebnisse aus Auswerteprotokoll B wiesen sowohl hinsichtlich der Endoleckdetektion als auch der -klassifikation keine Unterschiede zu den Ergebnissen aus Protokoll A auf. In Protokoll C wurden alle in Gruppe A identifizierten Endolecks erkannt $\left(r_{p}=100 \%\right)$. Darüber hinaus wurden mit der venösen Phase jedoch insgesamt 5 zusätzliche Endolecks diagnostiziert. In 3 Fällen wurde das Endoleck nur von einem Untersucher detektiert

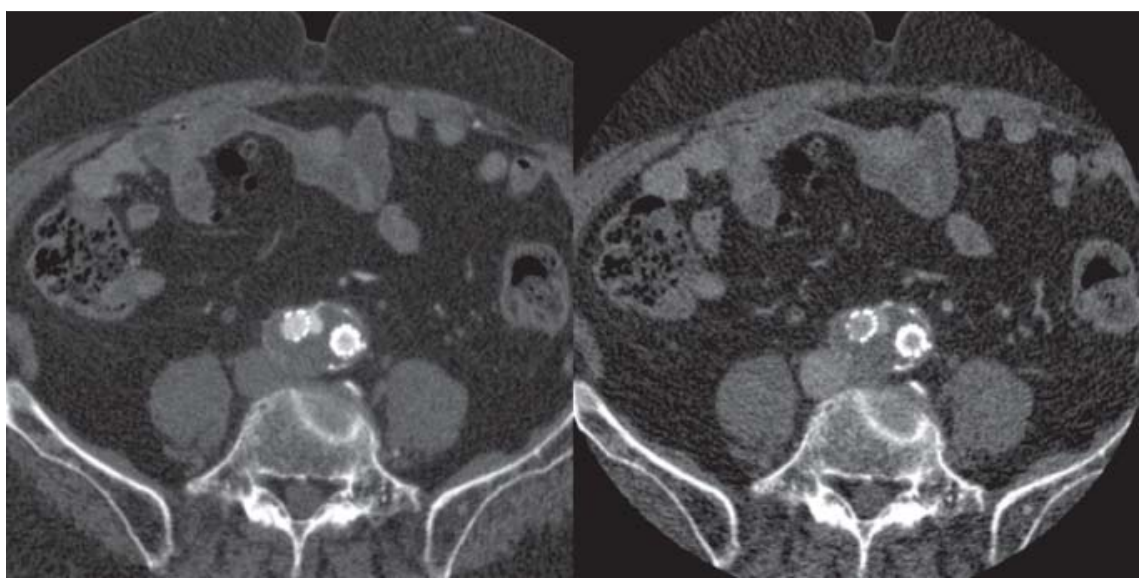

Abb. 3 Arterieller Datensatz (links) und Niedrigenergie-Datensatz aus der venösen Phase (rechts). Kleines Typ-II-Endoleck angrenzend an den rechten Prothesenschenkel des Endografts. Der Kontrastmittelpool lässt sich in beiden Bildserien ähnlich gut darstellen. Im Niedrigenergiebild liegt jedoch ein deutlich erhöhtes Bildrauschen vor. 
und vom dritten Auswerter ausgeschlossen. In allen Fällen wurde ein Typ 2 Endoleck vermutet. In 2 Fällen wurde das Vorliegen eines Typ-II-Endolecks vom dritten Auswerter bestätigt, sodass 2 falsch positive Befunde vorlagen (3,9\%). Bei einem der betroffenen Patienten lag ein BMI von 28,4 mit entsprechend erhöhtem Bildrauschen vor, der zweite Patient war normalgewichtig $(B M I=23,9)$. Hier lag ein fragliches Kontrastmitteldepot unterhalb der Gefäßwand der Aorta vor, das jedoch weder in der arteriellen Phase noch im Mischbild der venösen Phase eindeutig zu erkennen war ( $\bullet$ Abb.4). Der Befund wurde daher als falsch positiv interpretiert. Klinisch lag kein Hinweis auf ein Endoleck vor, der Aneurysmadurchmesser war im gesamten postoperativen Verlauf über 18 Monate stabil. Insgesamt ergab sich jedoch kein signifikanter Unterschied zum Referenzstandard $(p=0,125)$. Für dieses Protokoll lagen Sensitivität und negativ prädiktiver Wert bei $100 \%$, die Spezifität bei $94,1 \%$ und der positiv prädiktive Wert bei 89,5\% (๑ Tab.4).

In den Auswerteprotokollen A und B war die native Serie zum Ausschluss eines Endolecks in 11 (Reader 1) bzw. 12 (Reader 2) Fällen notwendig, wobei beide Auswerter die Notwendigkeit der Einbeziehung der nativen bzw. virtuell nativen Serie weitgehend übereinstimmend beurteilten $(\mathrm{K}=0,72)$. In Auswerteprotokoll C wurde die virtuell native Serie häufiger zur Beurteilung von Endolecks herangezogen (Reader 1 : $n=13$, Reader 2 : $n=15 ; k=0,71$ ), wobei dieser Unterschied nicht das Signifikanzniveau erreichte $(p=0,5)$.

\section{Effektive Dosis}

Die Scanlängen sowie die entsprechenden Dosiswerte für die unterschiedlichen Untersuchungsphasen sind aus $\bullet$ Tab. 4 ersichtlich. Das mittlere DLP betrug für die native Phase $302 \pm 107 \mathrm{mGy}$, für die arterielle Phase 475,20 $\pm 126,25 \mathrm{mGy}$ und für die venöse Phase $467,59 \pm 131,47 \mathrm{mGy}$.

Daraus ergibt sich eine durchschnittliche effektive Dosis für den Nativscan von 4,53 $\pm 1,61 \mathrm{mSv}$, für die arterielle Phase von 7,13 $\pm 1,89 \mathrm{mSv}$ und für die venöse Phase von 7,01 $\pm 1,97 \mathrm{mSv}$.

Für die Auswerteprotokoll A zugrunde liegenden Untersuchungsserien ergab sich eine effektive Dosis von 18,67 $\pm 5,24$ mSv. Für Protokoll B lag die effektive Dosis bei 14,14 $\pm 3,83 \mathrm{mSv}$ und für Protokoll C bei 7,01 $\pm 1,97 \mathrm{mSv}$ ( $\bullet$ Tab. 5).

Die Strahlenexposition für Protokoll C war somit um 62,45\% niedriger als für Protokoll A. Die Unterschiede hinsichtlich der effektiven Dosis der den 3 Auswerteprotokollen zugrundeliegenden Untersuchungsprotokolle waren signifikant $(p<0,001)$.

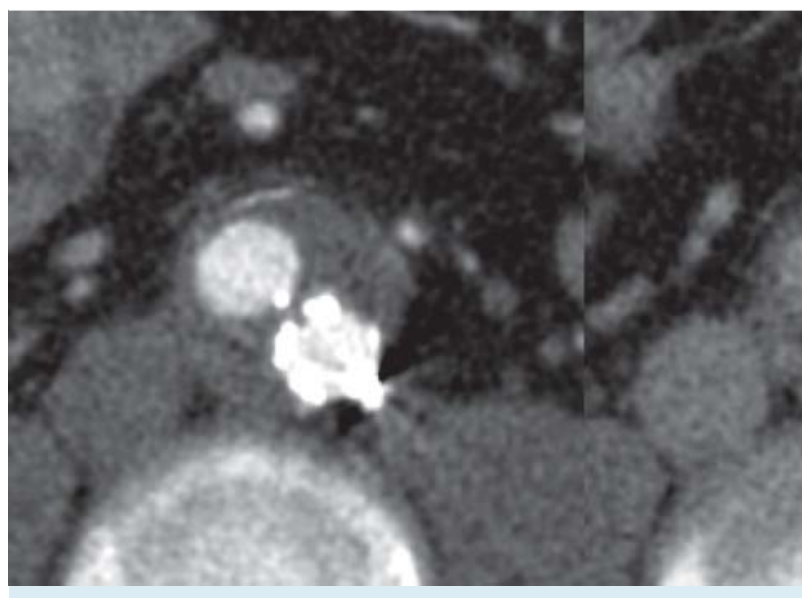

Abb. 4 Arterieller Datensatz (links), Mischbild der venösen Phase (Mitte) und Niedrigenergie-Datensatz aus der venösen Phase (rechts). Im Niedrigenergie-Datensatz der venösen Phase wurde ein Typ-II-Endoleck diagnostiziert (Pfeil). Dieses ist jedoch weder in der arteriellen Phase noch im Misch-

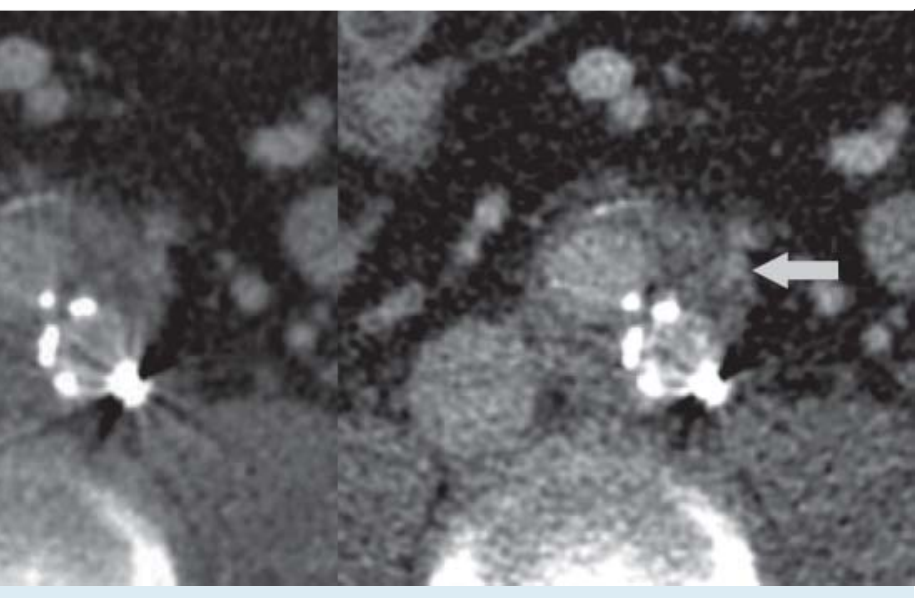

bild der venösen Phase eindeutig zu erkennen. Auch klinisch lag kein Hinweis auf ein Endoleck vor. Der Befund wurde deshalb als falsch positiv interpretiert. Im Niedrigdosisbild ist das Bildrauschen deutlich stärker ausgeprägt als in den Mischbildern der arteriellen und venösen Phase.

Tab. 4 Sensitivität, Spezifität, positiv und negativ prädiktiver Wert für die Erkennung von Endoleckagen im Auswerteprotokoll C im Vergleich zum Referenzstandard. In Klammern: 95 \%-Konfidenzintervall. PPV: positiv prädiktiver Wert; NPV: negativ prädiktiver Wert.

\begin{tabular}{|llll} 
& Sensitivität(\%) & Spezifität (\%) & PPV (\%) \\
\hline Protokoll C & $100(80,5-100)$ & $94,1(80,5-100)$ & $89,5(66,9-98,7)$ \\
\hline $\mathrm{n}$ & $(17 / 17)$ & $(32 / 34)$ & $(17 / 19)$
\end{tabular}

\begin{tabular}{|llll|} 
& native Phase & arterielle Phase & venöse Phase \\
\hline Scanlänge (mm) & $219 \pm 37$ & $468 \pm 33$ & $468 \pm 33$ \\
\hline CTDlvol (mGy) & $10,94 \pm 3,3$ & $9,61 \pm 2,37$ & $9,60 \pm 2,37$ \\
\hline DLP $(m G y)$ & $302 \pm 107$ & $475,20 \pm 126,25$ & $467,59 \pm 131,47$ \\
\hline Dosiseff $(m S v)$ & $4,53 \pm 1,61$ & $7,13 \pm 1,89$ & $7,01 \pm 1,97$ \\
\hline & Protokoll A & Protokoll B & Protokoll C \\
\hline Dosiseff $(m S v)$ & $18,67 \pm 5,24$ & $14,14 \pm 3,83$ & $7,01 \pm 1,97$ \\
\hline
\end{tabular}

Tab. 5 Übersicht der relevanten Dosisparameter sowie der effektiven Dosen für die native, arterielle und venöse Phase und die unterschiedlichen Auswerteprotokolle (Mittelwert \pm Standardabweichung). 


\section{Diskussion}

$\nabla$

Zur postinterventionellen CT-Langzeitkontrolle nach EVAR sieht das aktuelle Standardverfahren ein Drei-Phasen-CT vor, bestehend aus Nativscan sowie arterieller und venöser Phase $[8,19]$. Die Ergebnisse dieser Studie zeigen, dass ein Protokoll aus virtuellen Nativbildern und $80 \mathrm{kV}$-Bildern der venösen Phase eine zuverlässige Alternative zum Standardprotokoll darstellt. Alle im Standardverfahren erkannten Endolecks wurden korrekt identifiziert. Zudem konnte die Strahlenexposition gegenüber dem Standardverfahren um 62,45\% reduziert werden.

Die Strahlenexposition stellt insbesondere aufgrund der Notwendigkeit von Langzeitkontrollen die wichtigste Limitation der CT im Follow-up nach EVAR dar. Aktuelle Guidelines empfehlen zur Nachsorge nach EVAR auch weiterhin ein biphasisches CT nach Kontrastmittelgabe [21]. Rozenblit et al. haben dieses Verfahren durch einen Nativscan ergänzt und mit zwei Protokollen bestehend aus Nativscan und arterieller Phase bzw. arterieller und venöser Phase verglichen und kamen zu dem Schluss, dass ein Nativscan sowie die venöse Phase zur Identifizierung von Endolecks unverzichtbar sind [9]. Dabei dient der Nativscan in erster Linie der Lokalisation von Kalzifikationen. Iezzi et al. schlugen vor, den Nativscan einmalig bei der ersten postoperativen Untersuchung durchzuführen und diesen im Follow-up mit einer aktuellen CT-Angiografie zu kombinieren [7]. Die Verkalkungen der Aortenwand zeigen jedoch eine gewisse Dynamik, sodass dieses Verfahren insbesondere im Rahmen von Langzeitkontrollen zweifelhaft erscheint [22].

Das Dual-Energy-Verfahren ermöglicht die Berechnung von virtuellen Nativbildern aus einer Kontrastmittelserie, sodass auf die Durchführung einer echten Nativserie verzichtet werden kann. Unsere Ergebnisse zeigen, dass eine native Serie bei ca. 20\% der Patienten zur Beurteilung der Endolecks benötigt wird, wobei sich zwischen den Auswerteprotokollen A und B keine Unterschiede hinsichtlich der Erkennung und Klassifikation von Endolecks ergaben und somit virtuelle und reelle Nativbilder als gleichwertig gelten dürfen. Stolzmann et al. berichten von einem falsch positiven Befund in der virtuellen Nativserie bei einem Patienten, bei dem das CT unmittelbar nach der Stentimplantation durchgeführt wurde und residuelles Kontrastmittel im Aneurysmasack fälschlicherweise als Endoleck interpretiert wurde. Die Autoren kommen aber wie Maturen et al. und Sommer et al. zu dem Schluss, dass die echte Nativserie durch die Berechnung virtuell nativer Bilder ersetzt werden kann [23 - 25]. Dadurch ließ sich eine Reduktion der Strahlenbelastung von nahezu $25 \%$ erreichen.

Neben der Reduktion der Untersuchungsphasen kann die Strahlenexposition der Patienten auch durch eine Reduktion der Röhrenspannung erzielt werden [26]. Dadurch kommt es zu einer relativen Dichteanhebung des Kontrastmittels. Iezzi et al. konnten zeigen, dass die Durchführung eines Standardprotokolls mit 80 statt $120 \mathrm{kV}$ bei einem gleichbleibenden Kontrast-Rausch-Verhältnis zu einer Reduktion der Strahlendosis um 74\% führt [27]. In der vorliegenden Studie wurde der $80 \mathrm{kV}$-Datensatz aus der venösen Phase ausgewertet. In dieser Phase können auch sogenannte „Low-flow“-Endolecks erkannt werden, welche in der arteriellen Phase oft nicht diagnostiziert werden. Es wird jedoch auch die Meinung vertreten, dass die arterielle Phase zur Erkennung von Endoleckagen ausreichend ist, da „Low-flow“-Endolecks nicht therapiebedürftig sind [28]. In unserer Studie wurden alle Endolecks auch im $80 \mathrm{kV}$-Datensatz aus der venösen Phase erkannt. Zusätzlich wurden 2 Fälle als falsch positiv bewertet, wobei einer der Patienten übergewichtig war und entsprechend eine unterdurchschnittliche Bildqualität vorlag. In beiden Fällen wurde ein Typ-II-Endoleck mit langsamem retrogradem Fluss durch Kollateralarterien vermutet. Im 80kV-Datensatz liegt insbesondere bei Patienten mit einem großen Durchmesser ein im Vergleich zur Standardbildgebung deutlich erhöhtes Bildrauschen vor [27]. Zusätzlich sind die durch die Stentstreben verursachten Aufhärtungsartefakte stärker ausgeprägt. Dadurch ist die Bildqualität im Vergleich zum Standardverfahren etwas eingeschränkt. Unsere Ergebnisse decken sich insgesamt mit der Arbeit von Stolzmann et al., die ebenfalls von einem falsch positiven Befund aufgrund erhöhten Bildrauschens bei einem übergewichtigen Patienten berichten [22].

Durch den Verzicht auf die native und arterielle Phase konnte die Strahlenexposition der Patienten gegenüber dem Standardverfahren um etwas mehr als $62 \%$ reduziert werden, was der Dosisreduktion in den Studien von Stolzmann et al. und Chandarana et al. entspricht $[16,23]$.

Bley et al. konnten zeigen, dass Endolecks auch mithilfe einer 3D-Volumetrie aus nativen Bilddaten anhand der Änderung des Volumens des Aneurysmasacks ausgeschlossen werden können. Dies ermöglicht für die Nachsorge den Verzicht auf die arterielle und venöse Phase und damit eine Reduktion der Strahlenexposition um bis zu 82\% [29]. Bei einem Anstieg des Aneurysmavolumens müssen die Kontrastmittelphasen dann allerdings nachgeholt werden, was jedoch nur bei $9 \%$ der Patienten der Fall war. Neben der CT stehen auch andere Verfahren zur Bildgebung nach EVAR zur Verfügung [8]. Insbesondere die Kontrastsonografie (CEUS) hat sich mittlerweile als zuverlässiges Verfahren zur Nachsorge etabliert. Im Vergleich zur Doppler-Sonografie können Endolecks deutlich besser detektiert werden [30]. Die Kontrastierung auch kleiner Gefäße in Verbindung mit der dynamischen Flussinformation ermöglicht die zuverlässige Erkennung von Endolecks. Einige Arbeiten konnten auch eine Überlegenheit der CEUS gegenüber der CT-Angiografie zeigen [30-33]. Einschränkend ist jedoch zu erwähnen, dass die verwendeten CTProtokolle teilweise die Möglichkeiten der CT nicht voll ausschöpfen. Die CEUS setzt zudem viel Erfahrung beim Untersucher voraus, in einigen Fällen ist die Beurteilbarkeit der Aorta bei übergewichtigen Patienten oder Darmgasüberlagerung nur eingeschränkt möglich. Die aktuellen Daten zeigen jedoch, dass der CEUS bei fehlendem primärem Nachweis eines Endolecks eine zuverlässige Alternative zur CT zur Nachsorge nach EVAR darstellt. Hinsichtlich der Diagnose von Endograftmigration und morphologischen Veränderungen ist die CT der Sonografie überlegen, sodass der Einsatz der CT weiterhin indiziert ist [32, 34].

\section{Limitationen und Schlussfolgerung}

In der vorliegenden Studie wurden sowohl die arterielle als auch die venöse Phase als Dual-Energy-Untersuchung gefahren. Dadurch war das Bildrauschen auch im Mischbild der arteriellen Phase im Vergleich zu einer normalen $120 \mathrm{kV}$-Akquisition etwas erhöht, was jedoch subjektiv nicht zu einer Einschränkung der Beurteilbarkeit der Schnittbilder geführt hat.

Eine weitere Limitation der Studie ist sicherlich die Anzahl der eingeschlossenen Patienten und die damit verbundene, relativ geringe Anzahl nachgewiesener Endolecks. Die Ergebnisse der vorliegenden Studie sollten daher vor allem hinsichtlich der Darstellbarkeit der weniger häufigen Endolecktypen in weiterführenden Studien validiert werden.

Darüber hinaus wurden in unserer Studie nur Patienten mit Endoleck-Typ I-III untersucht. Da grundsätzlich zwischen 5 Endo- 
leck-Typen unterschieden wird, bleibt zu prüfen, ob Typ IV und V mittels Protokoll C ebenso sicher identifizierbar sind.

Trotz dieser Einschränkungen stellt ein Protokoll aus virtuellen Nativbildern und 80 kV-Bildern der venösen Phase eine zuverlässige Alternative zur Detektion von Endolecks nach EVAR dar und ermöglicht eine Reduktion der Strahlenexposition gegenüber dem Standardverfahren von über $60 \%$.

\section{Literatur}

1 Parodi JC, Palmaz JC, Barone HD. Transfemoral intraluminal graft implantation for abdominal aortic aneurysm. Ann Vasc Surg 1991; 5: $491-499$

2 Mestres G, Zarka ZA, Garcia-Madrid C et al. Early abdominal aortic endografts: a decade follow-up results. Eur J Vasc Endovasc Surg 2010; 40: $722-728$

3 Quinney BE, Parmar GM, Nagre SB et al. Long-term single institution comparison of endovascular aneurysm repair and open aortic aneurysm repair. J Vasc Surg 2011; 54: 1592 - 1598

4 Greenhalgh RM, Brown LC, Powell JT. Endovascular versus Open Repair of Abdominal Aortic Aneurysm. N Engl J Med 2010; 362: 1863 - 1871

5 Zarins CK, White RA, Hodgson KL et al. Endoleak as a predictor of outcome after endovascular aneurysm repair: AneuRx multicenter clinical trial. J Vasc Surg 2000; 32: 90-107

6 Espinosa G, Alves MR, Caramalho MF et al. A 10-Year Single-Center Prospective Study of Endovascular Abdominal Aortic Aneurysm Repair With the Talent Stent-Graft. J Endovasc Ther 2009; 16: 125-135

7 Lezzi R, Cotroneo AR, Filippone A et al. Multidetector CT in abdominal aortic aneurysm treated with endovascular repair: Are unenhanced and delayed phase enhanced images effective for endoleak detection? Radiol 2006; 241: 915-921

8 Stavropoulos SW, Charagundla SR. Imaging techniques for detection and management of endoleaks after endovascular aortic aneurysm repair. Radiology 2007; 243: 641 - 655

9 Pache G, Euringer W, Siepe $M$ et al. CT-Angiografie zur prä- und postoperativen Evaluation in der thorakalen Aortenchirurgie. Fortschr Röntgenstr 2011; 183: 334-346

10 Rozenblit AM, Patlas M, Rosenbaum AT et al. Detection of endoleaks after endovascular repair of abdominal aortic aneurysm: value of unenhanced and delayed helical CT acquisitions. Radiol 2003; 227: 426-433

11 Kalef-Ezra JA, Karavasilis S, Ziogas D et al. Radiation burden of patients undergoing endovascular abdominal aortic aneurysm repair. J Vasc Surg 2009; 49: $283-287$

12 Flohr TG, McCollough CH, Bruder H et al. First performance evaluation of a dual-source CT (DSCT) system. Eur Radiol 2005; 16: 256 - 268

13 Johnson TRC, Krauß B, Sedlmair $M$ et al. Material differentiation by dual energy CT: initial experience. Eur Radiol 2007; 17: 1510-1517

14 Maturen KE, Kaza RK, Liu PS et al. "Sweet Spot" for Endoleak Detection: Optimizing Contrast to Noise Using Low keV Reconstructions From Fast-Switch kVp Dual-Energy CT. J Comput Assist Tomogr 2012; 36: $83-87$

15 Avrin DE, Macovski A, Zatz LE. Clinical application of Compton and photo-electric reconstruction in computed tomography: preliminary results. Invest Radiol 1978; 13: 217-222

16 Chandarana H, Godoy MCB, Vlahos I et al. Abdominal Aorta: Evaluation with Dual-Source Dual-Energy Multidetector CT after endovascular repair of aneurysms - Initial observations. Radiol 2008; 249: 692 -700
17 Pitton MB, Schmiedt $W$, Neufang $A$ et al. Klassifikation und Therapie von Endolecks nach endovaskulärer Behandlung von abdominellen Aortenaneurysmen. Fortschr Röntgenstr 2005; 177: 24-34

18 Menzel HG, Schibilla H, Teunen D. European guidelines on quality criteria for computed tomography. Publication No. EUR 16262 EN. Luxembourg: European Commission; 2000

19 Lenzen $H$. Medizinphysiker in der Deutschen Röntgengesellschaft Fortschr Röntgenstr 2012; 184: 987-991

20 Landis $L R$, Koch GG. The measurement of observer agreement for categorical data. Biometrics 1977; 33: 159-174

21 Moll FL, Powell JT, Fraedrich G et al. Management of Abdominal Aortic Aneurysms Clinical Practice Guidelines of the European Society for Vascular Surgery. Eur J Vasc Endovasc Surg 2011; 41: S1 - S58

22 Adler Y, Fisman EZ, Shemesh J et al. Spiral computed tomography evidence of close correlation between coronary and thoracic aorta calcifications. Atherosclerosis 2004; 176: 133-138

23 Stolzmann P, Frauenfelder T, Pfammatter T et al. Endoleaks after Endovascular Abdominal Aortic Aneurysm Repair: Detection with DualEnergy Dual-Source CT. Radiology 2008; 249: 682 - 691

24 Maturen KE, Kleaveland PA, Kaza RK et al. Aortic Endograft Surveillance: Use of Fast-Switch kVp Dual-Energy Computed Tomography With Virtual Noncontrast Imaging. J Comput Assist Tomogr 2011; 35: 742 746

25 Sommer WH, Graser A, Becker CR et al. Image Quality of Virtual Noncontrast Images Derived from Dual-energy CT Angiography after Endovascular Aneurysm Repair. J Vasc Interv Radiol 2010; 21: 315-321

26 Pache $G$, Euringer $W$, Siepe $M$ et al. CT Angiography for the pre- and postoperative evaluation of the thoracic aorta. Fortschr Röntgenstr 2011; 183: 334-346

27 Iezzi R, Cotroneo AR, Giammarino A et al. Low-dose multidetector-row CT-angiography of abdominal aortic aneurysm after endovascular repair. Eur J Radiol 2011; 79: 21 - 28

28 Hong C, Heiken JP, Sicard GA et al. Clinical significance of endoleak detected on follow-Up CT after endovascular repair of abdominal aortic aneurysm. Am J Roentgenol 2008; 191: 808-813

29 Bley TA, Chase PJ, Reeder SB et al. Endovascular abdominal aortic aneurysm repair: nonenhanced volumetric CT for follow-up. Radiol 2009; 253: $253-262$

30 Cantisani V, Ricci P, Grazhdani H et al. Prospective comparative analysis of colour-Doppler ultrasound, contrast-enhanced ultrasound, computed tomography and magnetic resonance in detecting endoleak after endovascular abdominal aortic aneurysm repair. Eur J Vasc \& Endovasc Surg 2011; 41: 186-192

31 Perini P, Sediri I, Midulla $M$ et al. Single-centre prospective comparison between contrast-enhanced ultrasound and computed tomography angiography after EVAR. Eur J Radiol 2011; 42: 797-802

32 Carrafiello G, Recaldini C, Laganà D et al. Endoleak detection and classification after endovascular treatment of abdominal aortic aneurysm: value of CEUS over CTA. Abdom Imaging 2008; 33: 357-362

33 Henao EA, Hodge MD, Felkai DD et al. Contrast-enhanced Duplex surveillance after endovascular abdominal aortic aneurysm repair: Improved efficacy using a continuous infusion technique. J Vasc Surg 2006; 43: 259-264

34 Mirza TA, Karthikesalingam A, Jackson D et al. Duplex Ultrasound and Contrast-Enhanced Ultrasound Versus Computed Tomography for the Detection of Endoleak after EVAR: Systematic Review and Bivariate Meta-Analysis. Eur J Vasc Endovasc Surg 2010; 39: 418-428 